\title{
Insight on (per)chlorate production, degradation and transport: Relevance to Mars
}

\author{
A. GURE ${ }^{1 *}$, T.A. SORENSON ${ }^{1,2}$, J.C. DEWEY ${ }^{1}$, \\ C.M. EGGLESTON ${ }^{1,3}$, B.A. PARKINSON ${ }^{2,3}$
}

${ }^{1}$ Dept. of Geology and Geophysics, ${ }^{2}$ Dept. of Chemistry, ${ }^{3}$ School of Energy Resources, University of Wyoming, Laramie, WY, USA (Correspondence: agure@uwyo.edu)

The presence of perchlorate $\left(\mathrm{ClO}_{4}^{-}\right)$on Mars indicates that strongly oxidizing processes occur on Mars' surface despite the dry, cold, and oxygen-deficient conditions. We showed that illuminated nanocrystalline powder or single crystals of semiconducting minerals can be effective catalysts in the photochemical production and degradation of $\mathrm{ClO}_{4}^{-}$ and chlorate $\left(\mathrm{ClO}_{3}{ }^{-}\right)$[1,2]. The results indicate a photostationary state under Earth temperature and pressure conditions [1,2], and also emphasize the role of oxygen as an electron acceptor in $\mathrm{ClO}_{4}^{-}$generation under Mars-like conditions. Here, we continue to identify controls on (per)chlorate production from $\mathrm{Cl}^{-}$in the presence of illuminated semiconducting minerals under simulated Mars temperature, pressure, atmospheric and regolith composition. Results from experiments conducted in a Mars simulation chamber show that $\mathrm{ClO}_{4}{ }^{-}$in contact with illuminated $(\lambda=340$ $-405 \mathrm{~nm}$ ) titanium dioxide (anatase) powder can be reduced to $\mathrm{ClO}_{3}$ from a high initial concentration down to a nearsteady or photostationary state. Because chlorine chemistry can involve volatiles, we are using a residual gas analyser to detect and measure volatile chlorine species formed within the simulation chamber. The results provide evidence for the photochemical production and degradation of oxychlorines not only in bulk aqueous medium, but also under the apparently dry surface environment of present-day Mars. We are also applying XPS to the detection of oxidized $\mathrm{Cl}$ species at illuminated semiconducting mineral surfaces.

The physical removal of (per)chlorate from near-surface environment is an important aspect of oxychlorine cycling on Mars. The deliquescent nature of some oxychlorine salts, especially (per)chlorate salts [3], can allow for the transport of oxychlorine brines away from the surface into Mars' nearsurface environment. This can only occur under specific surface temperature and relative humidity conditions, resulting in the formation of subsurface pockets of perchlorate brines. Early results from experiments performed in a temperature-modulated vessel show transport of $\mathrm{ClO}_{4}^{-}$ brine through sections of Mars regolith simulant (silica dioxide) and ice cores. Further experiments are underway.

[1] Schuttlefield et al. (2011) J. Am. Chem. Soc., 133, 17,521-17,523. [2] Gure et al. (2019) ACS Earth Space Chem., 3, 10, 2171-2174. [3] Chevrier et al. (2009). Geophys. Res. Lett. 36, L10202, 1-6. 